\title{
Cardiovascular Magnetic Resonance In Hypertrophic Cardiomyopathy
}

\author{
Afonso Akio Shiozaki*, Raymond J. Kim**, José Rodrigues Parga*, Eduardo Marinho Tassi***, Edmundo Arteaga*, \\ Carlos Eduardo Rochitte* \\ *Cardiovascular Magnetic Resonance and Computed Tomography Sector of the Instituto do Coração of the Hospital das Clínicas - HCFMUSP \\ - São Paulo, SP **Duke Cardiovascular Magnetic Resonance Center, Durham, North Carolina - EUA ***Cardiovascular Magnetic Resonance and \\ Computed Tomography Sector of the Diagnósticos da America S.A.- Rio de Janeiro, RJ
}

Hypertrophic cardiomyopathy $(\mathrm{HCM})$ is the most frequent genetic cardiac disease that causes sudden death in young people, with an incidence of 1:500 adults.

The routinely used criteria for worst prognosis have limited sensitivity and specificity. Thus, the estimated risk of evolving to dilated cardiomyopathy or sudden death is somewhat inaccurate, leading to management uncertainty of HCM patients.

Therefore, an accurate noninvasive method for the diagnosis of $\mathrm{HCM}$ with prognostic value is of great importance.

In the last years, Cardiovascular Magnetic Resonance (CMR) emerged not only as a diagnostic tool, but also as a study with prognostic values, by characterizing myocardial fibrosis with great accuracy in HCM patients. Additionally, CMR identifies the types of hypertrophy, analyses the ventricular function, estimates the intraventricular gradient and allows the determination of differential diagnosis. Moreover, CMR can uniquely access myocardial fibrosis in HCM.

\section{Introduction}

Hypertrophic cardiomyopathy (HCM) is a genetic cardiac disease which is the major responsible for sudden death among youth ${ }^{1}$, with relatively frequent incidence of 1:500 in adults ${ }^{2}$.

The polygenic character of the disease with over 200 mutations ${ }^{3}$, leads to an extremely varied phenotypic expression $^{4}$. While some subjects remain asymptomatic for their whole lives, others present sudden death as the first presentation $^{5}$.

The common risk factors used to stratify patients are: cardiac sudden death and family history of $\mathrm{HCM}$; unexplained syncope; non-sustained ventricular tachycardia; abnormal blood pressure response during upright exercise testing in subjects $<40$ years old; and presence of severe left ventricular hypertrophy ( $>30 \mathrm{~mm}$ by echocardiogram). These criteria are very useful to physicians in guiding treatment of $\mathrm{HCM}$ subjects ${ }^{6}$.

\section{Key words}

Hypertrophic cardiomyopathy; magnetic resonance; diagnosis; prognosis.

Mailing Address: Carlos Eduardo Rochitte - InCor •

Setor de Ressonância Magnética e Tomografia Computadorizada Cardiovascular

Av. Dr. Enéas de Carvalho Aguiar, 44 - 05403-000 - São Paulo, SP - Brazil

E-mail: rochitte@incor.usp.br

Received for publication on May 23, 2006; revised manuscript received

June 18, 2006; accepted July 3, 2006
However, the accuracy of these criteria is limited, leading to uncertainty about the real risk of sudden death.

Therefore, it is crucially important to have new diagnostic methods with enough prognostic power to identify potential subjects with higher risk of sudden death and ventricular dilatation form. This approach would allow more aggressive therapy with this group of subjects.

In the last few years cardiovascular magnetic resonance (CMR) has emerged as a very accurate tool in the diagnosis of HCM, even being considered as the first choice exam ${ }^{7}$. CMR evaluates several hypertrophic patterns, the ventricular function, the pressure gradient between the left ventricular outflow tract and aorta and is extremely useful for the differential diagnosis with other entities. Besides, for the first time, a non-invasive method identifies and quantifies the distribution of myocardial fibrosis found in this disease ${ }^{8}$.

The role of cardiovascular magnetic resonance in hypertrophic cardiomyopathy

Recently the European Society of Cardiology (Consensus Panel Report ${ }^{7}$ and later the American College of Cardiology/ American Heart Association (Clinical Competence Statement on Cardiac Imaging with Magnetic Resonance) $)^{9}$ indicated CMR as first choice exam or at least equivalent to other diagnostic methods in the approach of several cardiomyopathies, including $\mathrm{HCM}$.

The excellent accuracy of CMR in anatomical and functional analysis of the left and right ventricles to quantify ventricular volume and mass ${ }^{10-12}$ has increased the sensitivity and specificity of the diagnosis of HCM (Figure1). This has allowed precise identification of several forms of hypertrophy (Figures 2, 3, 4 and 5) and determination of differential diagnosis.

A comparative study of two-dimensional echocardiograms and CMR among HCM subjects demonstrated the greater accuracy of CMR in diagnosing and differentiating several patterns of hypertrophy. In cases where LV hypertrophy is more accentuated on the free wall, the echocardiogram did not permit diagnosis of HCM and among subjects with confined hypertrophy in anterolateral wall underestimated the thickness of the wall in relation to $\mathrm{CMR}^{13}$ (Figures 4 and 5).

Two other studies demonstrated that echocardiograms have lower sensitivity compared to that of CMRs. In the diagnosis of apical hypertrophic cardiomyopathy, echocardiograms did not properly evaluate the apical segment in up to $40 \%$ of HCM subjects ${ }^{14,15}$ (Figure 2).

Recently, Petersen et $\mathrm{al}^{16}$ compared geometric LV indices by 


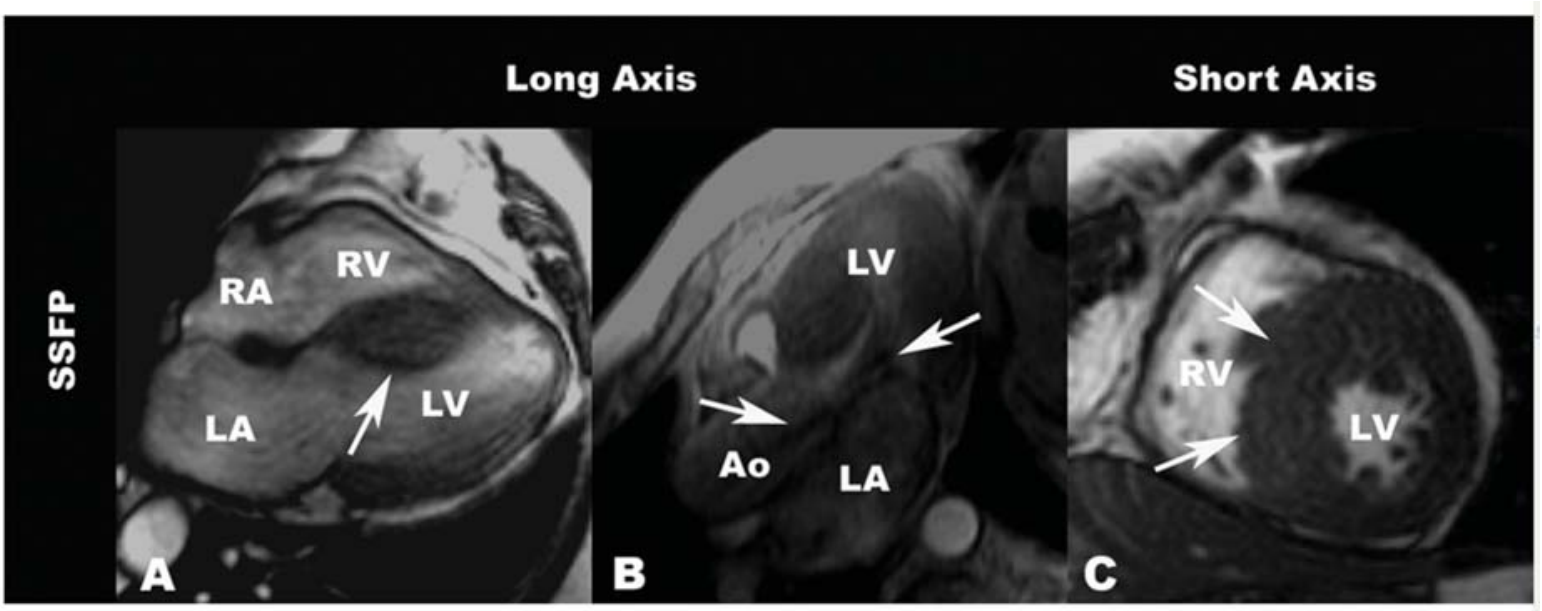

Fig. 1 - A Patient example with classic pattern of hypertrophic cardiomyopathy. Panel A shows a four chamber long axis view with a classic asymmetric septal hypertrophy (arrow). Panel B depicts LVOT view and flow artifacts (arrows) caused by pressure gradient. Panel C is a short axis view, showing asymmetric septal hypertrophy (compare thickness of septum and posterior wall). Ao - Aorta; LA - Left Atrium; LV - Left Ventricle; LVOT - Left Ventricle Outflow Tract; RA - Right Atrium; RV - Right Ventricle; SSFP - steady-state free precession.

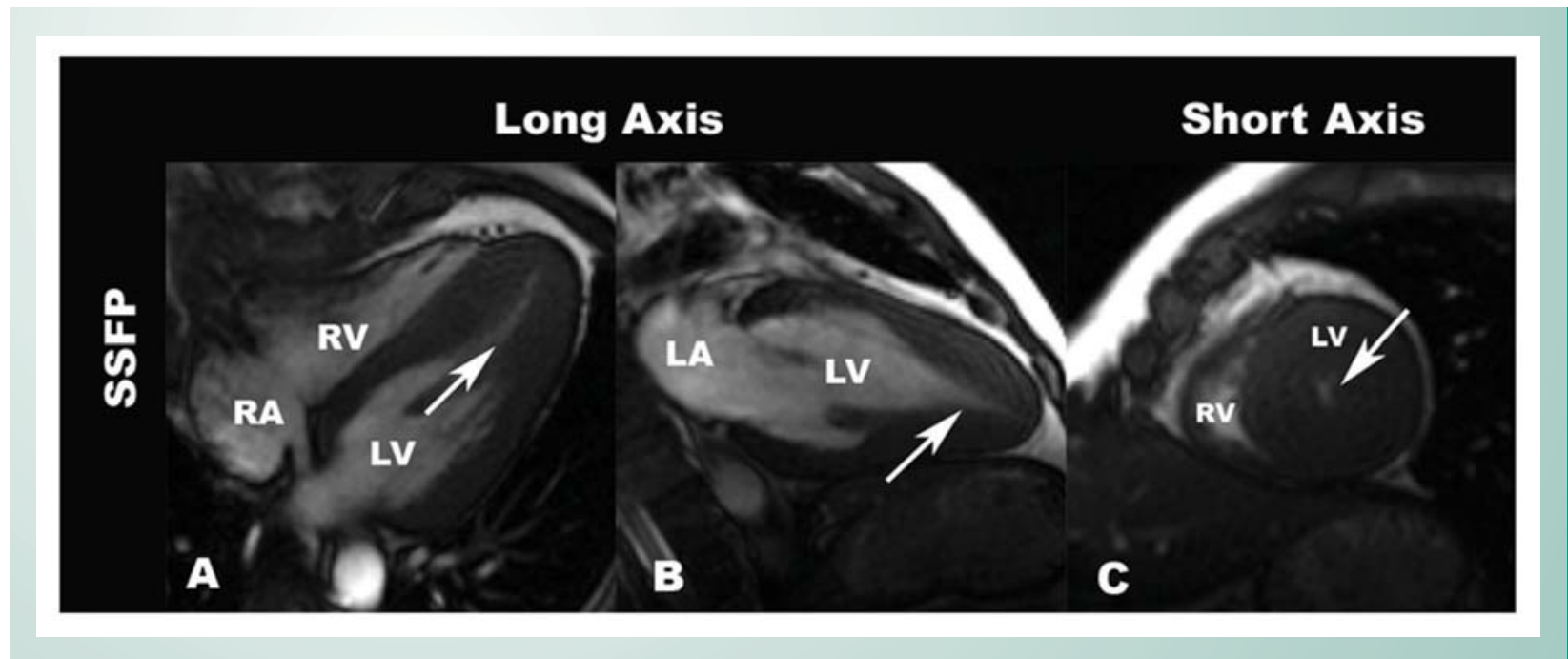

Fig. 2 - A Patient example with apical hypertrophic cardiomyopathy. Panels A and B shows long axis views with a apical regional hypertrophy, which produces a classic spade shape ventricular cavity in systole(arrows). In the short axis view (panel C), the apical cavity is almost obliterated (arrow). Ao - Aorta; LA - Left Atrium; LV - Left Ventricle; LVOT - Left Ventricle Outflow Tract; RA - Right Atrium; RV - Right Ventricle; SSFP - steady-state free precession.

CMR in HCM subjects, healthy volunteers, competitive athletes with physiologic hypertrophy, aortic stenosis and hypertensive heart disease. These authors pointed to the low sensitivity in the classic measurements used in diagnosis of HCM, such as the maximal end-diastolic wall thickness over $13 \mathrm{~mm}(40 \%$ sensitivity) and wall thickness ratio (28\% sensitivity). However, when a new geometric index was applied using the maximal end-diastolic thickness-to-left ventricular end-diastolic volume index ratio, the results were promising. They compared this index among athletes participating in high-level competitive sports, which were principally rowing, swimming, running, and cycling for at least the previous 18 months with an average of $19.2 \pm 6.8$ hours of training per week for the last $8.5 \pm 4.9$ years with that of the HCM subjects. This index for healthy volunteers and athletes was lower than $0.15 \mathrm{~mm} \times \mathrm{m}^{2} \times \mathrm{ml}$.

Indices higher than $0.15 \mathrm{~mm} \times \mathrm{m}^{2} \times \mathrm{ml}$ was observed in all pathological hypertrophies and presented a sensitivity of $80 \%$, specificity of $99 \%$, negative predictive value of $94 \%$, and positive predictive value of $95 \%$ in determining the differential diagnosis between HCM and the athletes' hearts ${ }^{16}$.

One of the major contributions of CMR, besides the anatomical and functional data and geometric indices, is the delineation of myocardial fibrosis in HCM, which follows specific patterns ${ }^{8}$. The presence of myocardial fibrosis increases even more the diagnostic accuracy and provide an insight on the prognosis. 


\section{Review Article}

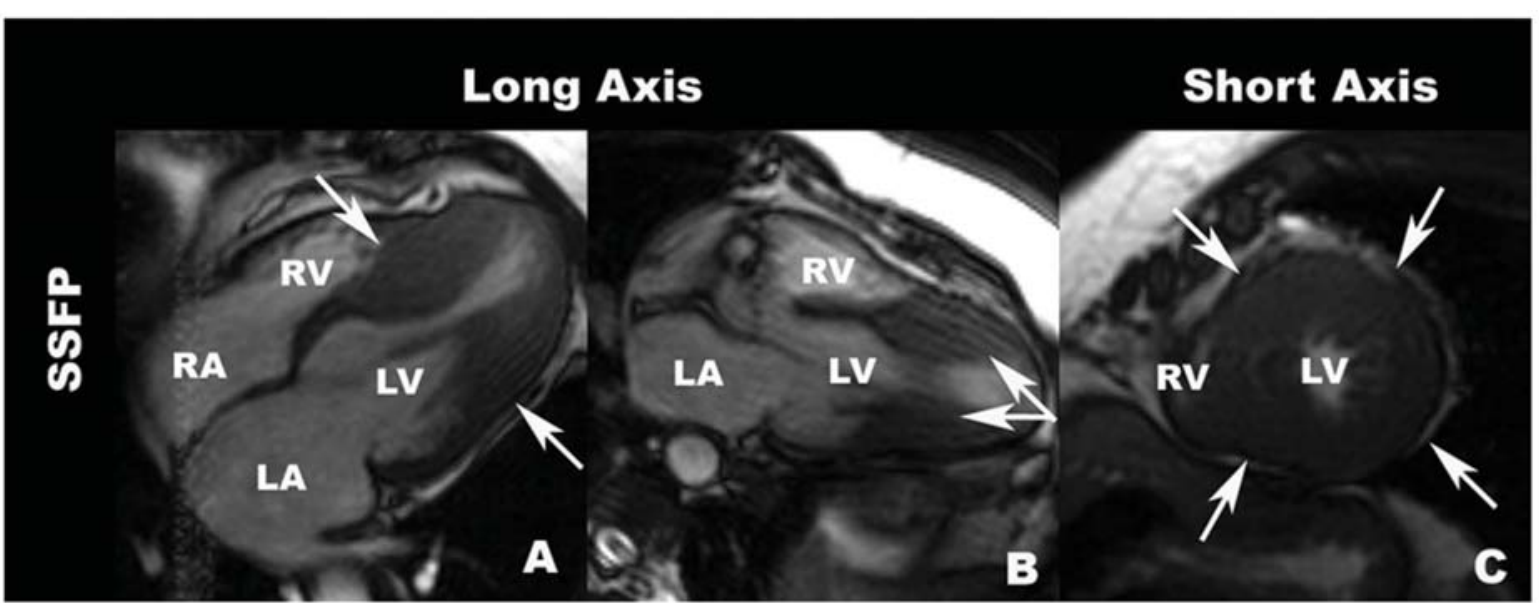

Fig. 3 - A Patient example with hypertrophic cardiomyopathy. Panels A and B. In the long axis views, there is isolated mid regional hypertrophy of left ventricular (arrows). Panel C. In the short axis view the circumferential mid LV hypertrophy can be clearly seen. Ao - Aorta; LA - Left Atrium; LV - Left Ventricle; LVOT - Left Ventricle Outflow Tract; RA - Right Atrium; RV - Right Ventricle; SSFP - steady-state free precession.

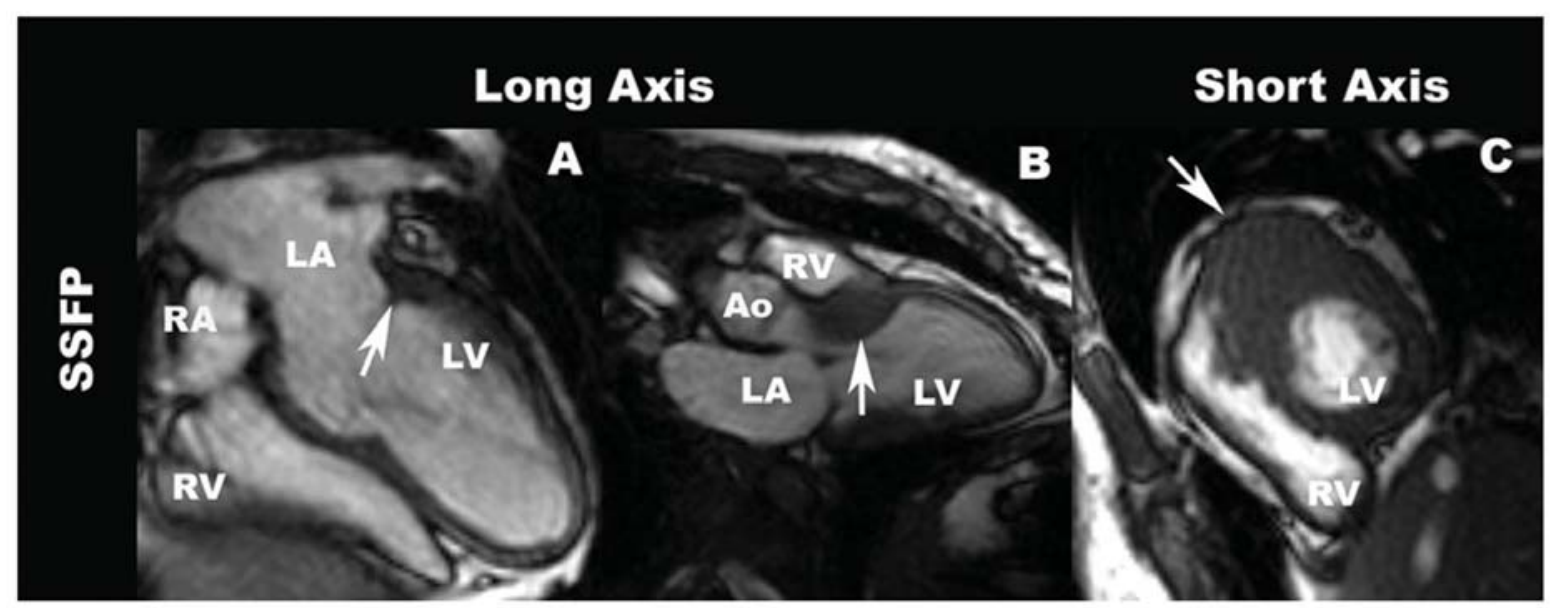

Fig. 4 - A Patient example with hypertrophic cardiomyopathy. Panel A: In the four chamber long axis view, one can not observe the wall hypertrophy. Panel B: In the LVOT view, observe the localized hypertrophy on basal segment of anterior left ventricular wall, causing dynamic obstruction of the left ventricular outflow tract (arrow). Panel C: In the short axis view, it is demonstrated the value of full LV coverage by contiguous slices from base to apex provided by CMR. Only in the short axis view the real dimension of the anterior basal localized hypertrophy can be uncovered. Ao - Aorta; LA - Left Atrium; LV - Left Ventricle; LVOT - Left Ventricle Outflow

Tract; RA - Right Atrium; RV - Right Ventricle; SSFP - steady-state free precession.

The technique that allows the identification of myocardial delayed enhancement (MDE) in ischemic heart disease was developed by Kim et al ${ }^{17-21}$. They demonstrated a close correlation between myocardial fibrosis by CMR and the fibrosis seen in histology ${ }^{22}$. Moreover, a landmark study by Kim et $\mathrm{a}^{23}$ defined a direct relationship between the extent of myocardial fibrosis by CMR and the potential for contractility recovery after revascularization. This data and other are the basis for considering CMR MDE as a gold-standard for myocardial viability in coronary artery disease ${ }^{24}$.

The MDE technique uses contrast based agents that have extracellular distribution and accentuate the difference in tissue relaxation characteristics between infarcted and normal myocardium. Following intravenous administration, the contrast agent diffuses rapidly from the intravascular to the extracellular compartment. Several minutes after injection, gadolinium in the normal myocardial, which has small extracellular space, washes out rapidly, while slow wash out occurs within areas with enlarged extracellular space, such as areas of myocardial fibrosis or necrosis ${ }^{17}$.

Choudhury et $\mathrm{al}^{8}$ were the first to use the MDE technique to detect myocardial fibrosis areas in $81 \%$ of asymptomatic or mild symptomatic HCM subjects. Furthermore, these authors observed that the delayed enhancement pattern was 


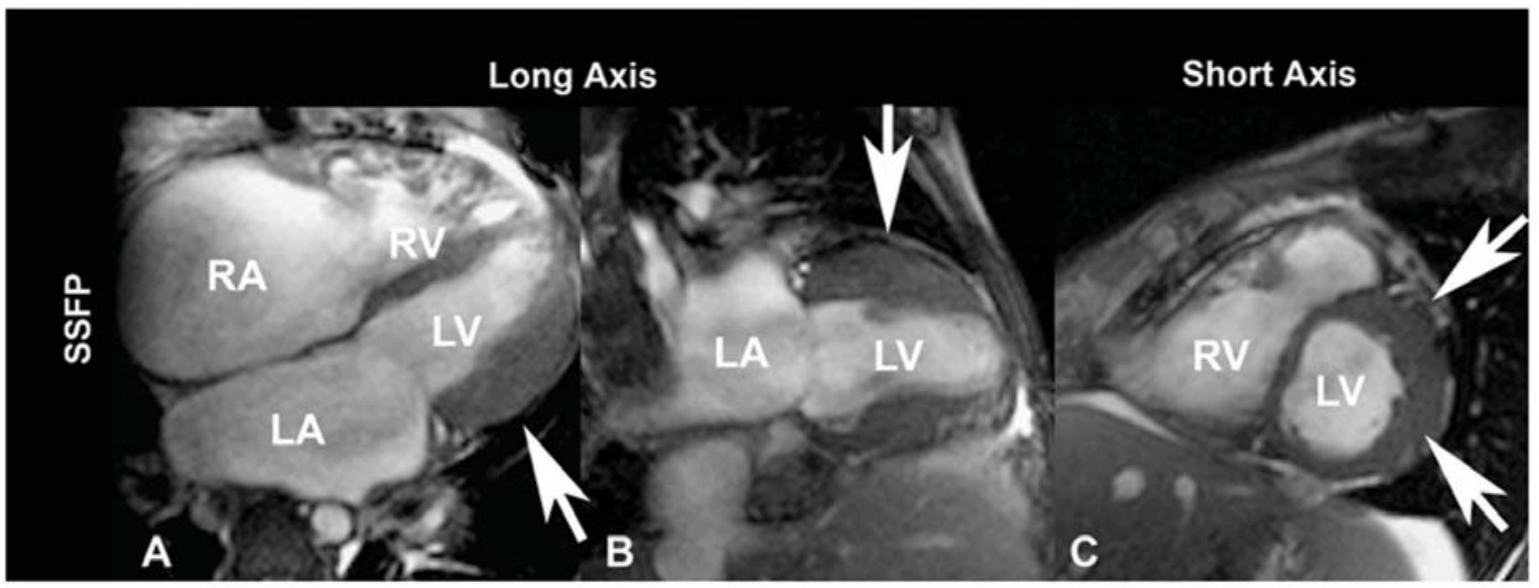

Fig. 5 - A Patient example with hypertrophic cardiomyopathy. In the four chamber long axis view, observe the lateral wall hypertrophy of left ventricular (arrow). In the three chambers long axis view, observe the anterior wall hypertrophy on medial-basal segment of anterior left ventricular wall (arrow). In the short axis view, the anterolateral hypertrophy of left ventricular wall can be evaluated simultaneously (arrows). Ao - Aorta; LA - Left Atrium; LV - Left Ventricle; LVOT - Left Ventricle Outflow Tract; RA - Right Atrium; RV - Right Ventricle; SSFP - steady-state free precession.

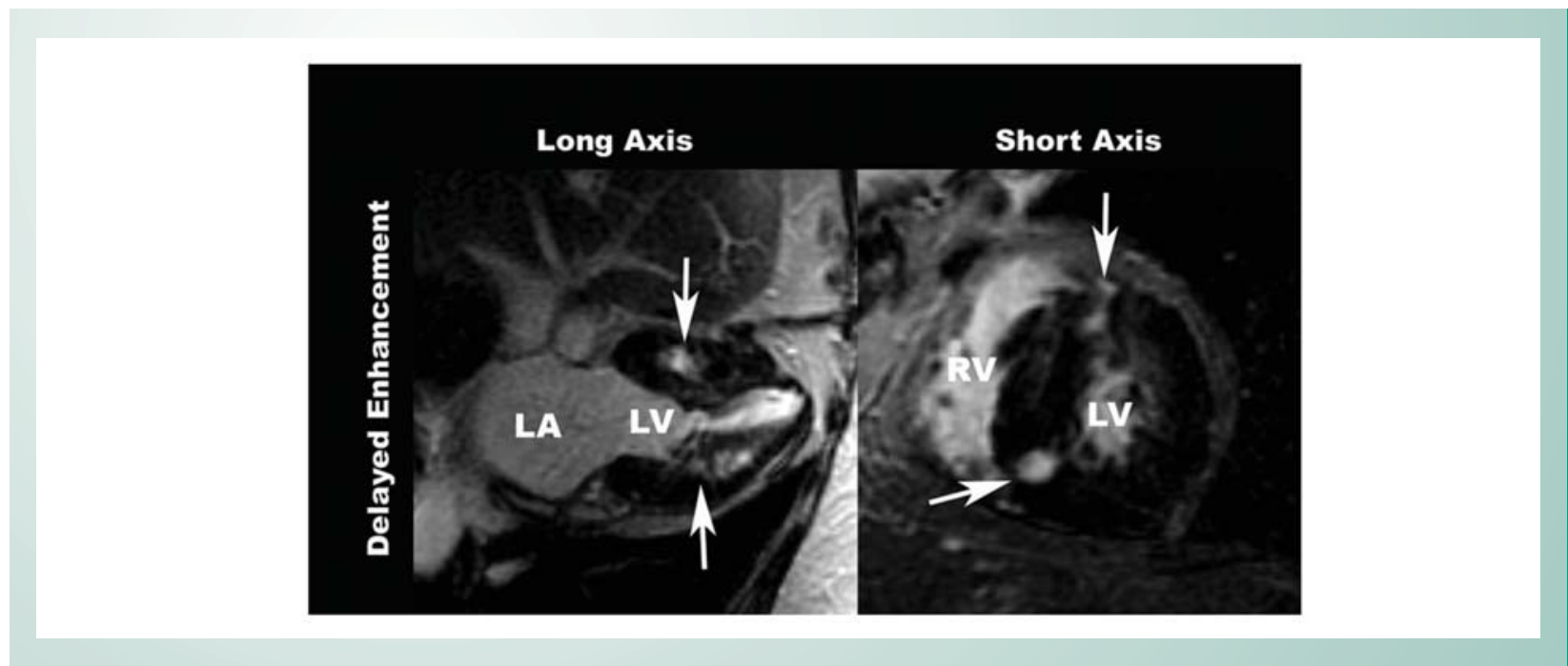

Fig. 6 - A Patient example with hypertrophic cardiomyopathy. Long (left) and short (right) axis view with multiple foci myocardial delayed enhancement with confluent pattern. In the short axis view it is observed the classic areas of delayed enhancement in HCM: in the junctions of interventricular septum and free wall right ventricle. This pattern of delayed enhancement can be visualized in about $80 \%$ of asymptomatic or mild symptomatic HCM subjects and is a more localized and homogeneous pattern. Ao - Aorta; LA - Left Atrium; LV - Left Ventricle; LVOT - Left Ventricle Outflow Tract; RA - Right Atrium; RV - Right Ventricle; SSFP - steady-state free precession.

very peculiar, with multiple foci standard. The myocardial hyperenhancement in HCM did not respect any specific coronary territory and in the majority of HCM subjects was found mostly at the junction between the interventricular septum and right ventricular free wall (Figure 6). This was previously demonstrated by HCM necropsy studies that showed myocardial disarray and highly accentuated fibrosis in these areas $^{8,24-26}$.

Once the fibrosis is visualized in the HCM by CMR, would the delayed enhancement have any prognostic implication?

In coronary artery disease, Bello et al ${ }^{27}$ showed that fibrosis measured by CMR in infarcted areas are better identifiers of patients who have a substrate for monomorphic ventricular tachycardia than LV ejection fraction.

Similar to ischemic cardiomyopathy, the probable origin of ventricular arrhythmias in HCM subjects ${ }^{28}$ is the multiple areas of electrical abnormal conduction caused by myocardial fibrosis, which could lead to sudden death ${ }^{29}$. Necropsy studies of HCM subjects demonstrated a higher fibrosis percentage in subjects who had sudden death when compared to those who died from non-cardiac causes ${ }^{30}$.

Moon et al, in $2003^{31}$, demonstrated the presence of delayed 


\section{Review Article}

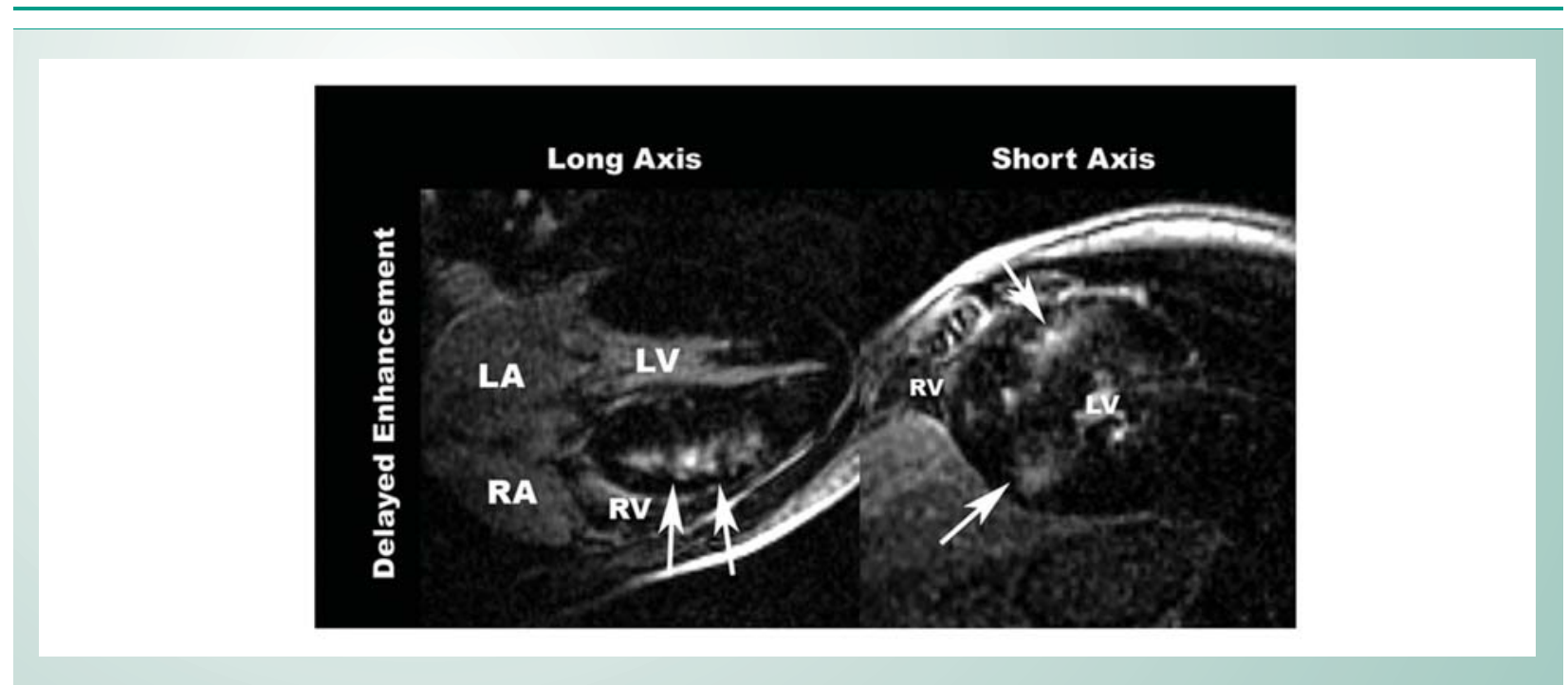

Fig. 7 - A Patient example with hypertrophic cardiomyopathy. Long (left) and short (right) axis view showing multiple foci delayed enhancement, with diffuse pattern. This diffuse and almost transseptal pattern is a more patchy and heterogeneous and is correlated with worse prognosis. Ao - Aorta; LA - Left Atrium; LV - Left Ventricle; LVOT - Left Ventricle Outflow Tract; RA - Right Atrium; RV - Right Ventricle; SSFP - steady-state free precession.

enhancement representing myocardial fibrosis areas by CMR in $79 \%$ of HCM patients. Previous studies demonstrated that sudden death in HCM correlates to a number of risk factors ${ }^{32}$. The percentage of delayed enhancement was significantly higher when patients presented more than 2 risk factors for sudden death $(15.7 \%$ vs. $8.6 \% p=0.02)$. It was also significantly higher in patients who presented with progressive disease, in the form of ventricular dilatation $(28.5 \%$ vs. $8.7 \%$ $p<0.001)$. This difference remained statistically significant when the subjects with more than 2 risk factors for sudden death or those with progressive dilated form were excluded.

These authors have also found the multiple foci delayed enhancement pattern previously related. Furthermore, they also observed 2 distinct patterns. The first pattern was denominated confluent hyperenhancement, a more localized and homogeneous pattern (Figure 6); the second, diffuse hyperenhancement, a more patchy and heterogeneous pattern (Figure 7). In the confluent pattern, there was a significant concentration of patients with lower risk for sudden death. (less than 2 risk factors), compared to the diffuse pattern, that was more frequent with higher risk (2 or more risk factors) for sudden death.

Thus, HCM subjects with diffuse delayed enhancement correlated to a higher percentage of fibrosis and to a worse prognosis for sudden death and left ventricular dilatation, while the HCM subjects with confluent delayed enhancement did not.

In conclusion, CMR provides a large amount of information on myocardial anatomy, function and tissue characterization that crucially improves HCM diagnosis. Of special importance is the singular CMR capability of detecting and quantifying myocardial fibrosis in HCM patients, which seems to be of great prognostic value.

Further studies will define if CMR can improve prediction of sudden death that will lead to changes in the current of management of HCM patients.

\section{References}

1. McKenna WJ, Firoozi S, Sharma S. Arrhythmias and sudden death in hypertrophic cardiomyopathy. Card Electrophysiol Rev. 2002; 6 (1-2): 26-31.

2. Maron BJ, Gardin JM, Flack JM, Gidding SS, Kurosaki TT, Bild DE. Prevalence of hypertrophic cardiomyopathy in a general population of young adults. Echocardiographic analysis of 4111 subjects in the CARDIA Study. Coronary Artery Risk Development in (Young) Adults. Circulation. 1995; 92 (4): 785-9.

3. Maron BJ, McKenna WJ, Danielson GK, Kappenberger LJ, Kuhn HJ, Seidman CE. American College of Cardiology/European Society of Cardiology Clinical Expert Consensus Document on Hypertrophic Cardiomyopathy. A report of the American College of Cardiology Foundation Task Force on Clinical Expert Consensus Documents and the European Society of Cardiology Committee for Practice Guidelines. Eur Heart J. 2003; 24 (21): 1965-91.

4. Maron BJ. Hypertrophic cardiomyopathy: a systematic review. JAMA. 2002; 287 (10): 1308-20.

5. Maron BJ, Casey SA, Poliac LC, Gohman TE, Almquist AK, Aeppli DM. Clinical course of hypertrophic cardiomyopathy in a regional United States cohort. JAMA. 1999; 281 (7): 650-5.

6. Maki S, Ikeda H, Muro A, Yoshida N, Shibata A, Koga Y. Predictors of sudden cardiac death in hypertrophic cardiomyopathy. Am J Cardiol. 1998; 82 (6): $774-8$

7. Pennell DJ, Sechtem UP, Higgins CB, Manning WJ, Pohost GM, Rademakers FE. Clinical indications for cardiovascular magnetic resonance (CMR): Consensus Panel report. Eur Heart J. 2004; 25 (21):1940-65.

8. Choudhury L, Mahrholdt H, Wagner A, Choi KM, Elliot MD, Klocke FJ. Myocardial scarring in asymptomatic or mildly symptomatic patients with hypertrophic cardiomyopathy. J Am Coll Cardiol. 2002; 40 (12): 2156-64.

9. Budoff MJ, Cohen MC, Garcia MJ, Hodgson JM, Hundley WG, Lima JA. ACCF/ AHA clinical competence statement on cardiac imaging with computed tomography and magnetic resonance: a report of the American College of 
Cardiology Foundation/American Heart Association/American College of Physicians Task Force on Clinical Competence and Training. J Am Coll Cardiol. 2005; 46 (2):383-402

10. Rajappan K, Bellenger NG, Anderson L, Pennell DJ. The role of cardiovascular magnetic resonance in heart failure. Eur J Heart Fail. 2000; 2 (3): 241-52

11. Heusch A, Koch JA, Krogmann ON, Korbmacher B, Bourgeois M. Volumetric analysis of the right and left ventricle in a porcine heart model: comparison of three-dimensional echocardiography, magnetic resonance imaging and angiocardiography. Eur J Ultrasound. 1999; 9 (3): 245-55

12. Bellenger NG, Francis JM, Davies CL, Coats AJ, Pennell DJ. Establishment and performance of a magnetic resonance cardiac function clinic. J Cardiovasc Magn Reson. 2000; 2 (1): 15-22.

13. Rickers C, Wilke NM, Jerosch-Herold M, Casey SA, Panse P, Panse N. Utility of cardiac magnetic resonance imaging in the diagnosis of hypertrophic cardiomyopathy. Circulation. 2005; 112 (6): 855-61.

14. Moon JC, Fisher NG, McKenna WJ, Pennell DJ. Detection of apical hypertrophic cardiomyopathy by cardiovascular magnetic resonance in patients with non-diagnostic echocardiography. Heart. 2004; 90 (6): 645-

15. Pons-Llado G, Carreras F, Borras X, Palmer J, Llauger J, Bayes de LA Comparison of morphologic assessment of hypertrophic cardiomyopathy by magnetic resonance versus echocardiographic imaging. Am J Cardiol. 1997; 79 (12): $1651-6$

16. Petersen SE, Selvanayagam JB, Francis JM, Myerson SG, Wilsmann F, Robson MD. Differentiation of athlete's heart from pathological forms of cardiac hypertrophy by means of geometric indices derived from cardiovascular magnetic resonance. J Cardiovasc Magn Reson. 2005; 7 (3): 551-8.

17. Kim RJ, Fieno DS, Parrish TB, Harris K, Chen EL, Simonetti O. Relationship of MRI delayed contrast enhancement to irreversible injury, infarct age, and contractile function. Circulation. 1999; 100 (19): 1992-2002.

18. Bucciarelli-Ducci C, Wu E, Lee DC, Holly TA, Klocke FJ, Bonow RO. Contrastenhanced cardiac magnetic resonance in the evaluation of myocardia infarction and myocardial viability in patients with ischemic heart disease. Curr Probl Cardiol. 2006; 31 (2): 128-68.

19. Fieno DS, Kim RJ, Chen EL, Lomasney JW, Klocke FJ, Judd RM. Contrastenhanced magnetic resonance imaging of myocardium at risk: distinction between reversible and irreversible injury throughout infarct healing. J Am Coll Cardiol. 2000; 36 (6): 1985-91.

20. Simonetti OP, Kim RJ, Fieno DS, Hillenbrand HB, Wu E, Bundy JM. An improved MR imaging technique for the visualization of myocardial infarction. Radiology. 2001; 218 (1): 215-23.

21. Rehwald WG, Fieno DS, Chen EL, Kim RJ, Judd RM. Myocardial magnetic resonance imaging contrast agent concentrations after reversible and irreversible ischemic injury. Circulation. 2002; 105 (2): 224-9.

22. Kim RJ, Judd RM. Gadolinium-enhanced magnetic resonance imaging in hypetrophic cardiomyopathy: in vivo imaging of the pathologic substrate for premature cardioac death? J Am Coll Cardiol 2003; 41(9): 1568-72.

23. Kim RJ, et al. The use of contrast-enhanced magnetic resonance imaging to identify reversible myocardial dysfunction. N Engl J Med. 2000; 343(20): 1445-53.

24. Basso C, Thiene G, Corrado D, Buja G, Melacini P, Nava A. Hypertrophic cardiomyopathy and sudden death in the young: pathologic evidence of myocardial ischemia. Hum Pathol. 2000; 31 (8): 988-98.

25. Kuribayashi T, Roberts WC. Myocardial disarray at junction of ventricular septum and left and right ventricular free walls in hypertrophic cardiomyopathy. Am J Cardiol. 1992; 70 (15): 1333-40.

26. Varnava AM, Elliott PM, Sharma S, McKenna WJ, Davies MJ. Hypertrophic cardiomyopathy: the interrelation of disarray, fibrosis, and small vessel disease. Heart. 2000; 84 (5): 476-82.

27. Bello D, Fieno DS, Kim RJ, Pereles FS, Passman R, Song G. Infarct morphology identifies patients with substrate for sustained ventricular tachycardia. J Am Coll Cardiol. 2005; 45 (7): 1104-8.

28. Hurwitz JL, Josephson ME. Sudden cardiac death in patients with chronic coronary heart disease. Circulation. 1992; 85 (Suppl 1): 143-149.

29. Maron BJ, Shen WK, Link MS, Epstein AE, Almquist AK, Daubert JP. Efficacy of implantable cardioverter-defibrillators for the prevention of sudden death in patients with hypertrophic cardiomyopathy. N Engl J Med. 2000; 342 (6): 365-73.

30. Varnava AM, Elliott PM, Mahon N, Davies MJ, McKenna WJ. Relation between myocyte disarray and outcome in hypertrophic cardiomyopathy. Am J Cardiol. 2001; 88 (3): 275-9.

31. Moon JC, McKenna WJ, McCrohon JA, Elliott PM, Smith GC, Pennell DJ. Toward clinical risk assessment in hypertrophic cardiomyopathy with gadolinium cardiovascular magnetic resonance. J Am Coll Cardiol 2003 May 7;41(9):1561-7.

32. Elliott PM, Poloniecki J, Dickie S, Sharma S, Monserrat L, Varnava A. Sudden death in hypertrophic cardiomyopathy: identification of high risk patients. J Am Coll Cardiol. 2000; 36(7): 2212-8 